\title{
Counting leaves without "finger-counting" by supervised multiscale frequency analysis of depth images from top view
}

Rousseau David
david.rousseau@univ-lyon1.fr

Van de Zedde HJ (Rick) rick.vandezedde@wur.nl

\author{
Université de Lyon, Laboratoire \\ CREATIS; CNRS, UMR5220; INSERM, \\ U1044, \\ Université Lyon 1; INSA-Lyon, Villeur- \\ banne, France \\ Wageningen UR Food Biobased \\ Research
}

Bornse Weilanden 9, 6708WG Wageningen, Netherlands

\begin{abstract}
Depth imaging is applied to characterize the shoot of seedlings from top-view. We demonstrate how quantitative informations of biological interest, such as leaves counting can be extracted from such images without performing 3D reconstruction of the shoot. This is obtained from 2D Fourier multiscale analysis without any requirement to segment nor detect leaves one by one numerically. We discuss the robustness and limitations of this approach and present possible extension with 3D Fourier analysis applied to estimate the plant plastochrone or $3 D+T$ Fourier analysis in the estimation of circadian rythms.
\end{abstract}

\section{Introduction}

Counting leaves of seedlings from top view is an important task for plant phenotyping. From a biological point of view, the number of leaves is a key marker of the growth stage of the plant. For technological concerns, the top view acquisition setup when coupled to conveyors of plants or camera allow the access to high throughput non invasive screening of large population of plants required in plant phenotyping. Counting leaves of seedlings from top view also constitutes a challenge for computer vision since the shoots of plants are, in general, rather homogeneous in color and leaves are poorly contrasted with each others with standard RGB or gray level luminance cameras. In monoaxial plants like seedlings, leaves are positioned sequentially along the main axis, with different heights and orientations. This disposition creates a strong spatial contrast between each leaves along the main axis of the seedling. 3D laser scanners or X-ray tomographs make it possible to record a full 3D acquisition and reconstruction of entire plants. However, when applied to large populations of plants, full 3D acquisitions can be time expensive for high-throughput phenotyping and will also produce huge amounts of data. In some biological contexts, full reconstruction of entire plants may not be necessary to characterize specific aspects of the morphology such 
as the sole counting of the number of leaves [ $[$ ]. In such cases, depth cameras have recently been shown to be useful tools (see [ [ ] for a recent review). Depth cameras produce 3D point clouds or depth maps where each pixel has an intensity proportional to the distance between the shoot of the plant and the camera. Depth cameras have been demonstrated to be useful for leaf segmentation, leaf orientation and canopy characterization.

In this report, we demonstrate that the counting of the leaves on seedlings can be obtained without having to perform the segmentation and recognition of each individual leaf. This is obtained with a supervised classification based on multiscale frequency analysis computed on depth images of seedlings from one single top view.

\section{Multiscale frequency analysis}

A classical approach to characterize the spatial frequency content of an image $I(x, y)$ consists in computing $F(I(x, y))$, its Fourier transform, followed by its power spectrum $P=$ $|F(I(x, y))|^{2}$, which is averaged along all the angular directions in a polar diagram, to produce a function of the spatial frequency $f$ called average power spectrum

$$
\operatorname{DSP}(f)=\frac{1}{2 \pi} \int_{0}^{2 \pi} P(f \exp (i \theta)) d \theta .
$$

A common signature of such analysis applied to natural landscapes with plants is scalefree power-law evolutions present in the frequency spectrum of luminance images [ $\square, \square]$ ]. Such power-law evolutions can be identified on a log-log graph when the average power spectrum follows a line over a large range of spatial frequencies. This corresponds to a power spectrum $D S P(f) \sim f^{\alpha}$ governed by a constant slope $\alpha$ across the frequency scales. Self-similarity accross scales, i.e. fractal features, has been reported in various properties of images from plants. The colorimetric organization of natural landscapes with plants also demonstrates self-similarity and fractal properties [ $\mathbf{\square}, \mathbf{Q}, \mathbf{Q}$, with other metrics based on the 3D RGB histogram. More recently, multiscale analysis has been undertaken for plant images obtained from another imaging technique delivering depth images of a physical scene [0]. The depth map images from outdoor scenes of woods and plants as in $[\square, \square]$ with the $D S P(f)$ of Eq. (1) were shown in [0] to also reveal self-similarity and fractal properties.

The interest of such multiscale image analyses revealing and characterizing fractal properties in plants are usually argued (see [] for a recent review), as important to contribute to the understanding of statistical properties of outdoor natural scenes since they have formed for a long period of time the environment in which the visual system has evolved and developed. From a biological point of view, the multiscale organization of plants which leads to high surface areas at the interfaces with the environment, ensuring for the plant efficient capture of nutrients and energy has a direct impact on their functioning, for instance for efficient interactions with their environment $[\varangle, \square, \square]$. Also, fractal characterization of plants is useful to devise synthetic models of plants with sufficient realism[四]. This is specially the case with the so-called dead leaves model which takes inspiration from the foliage of plants, with leaves of different sizes and illumination which are reproduced at various scales with occlusions $[$ 日, $\square]$. In this report, we will not explore these rather fundamental approaches in plant sciences and we focus on the demonstration of the practical interest of Fourier multiscale analysis for the informational task of leaf counting. 


\section{Depth images of seedlings from top view}

The type of plants used for the experiments carried in this report are tomato seedlings chosen at various stages of development classified as a function of the number of leaves accessible from top view starting with two up till six leaves. A set of depth images from top view of some of these different stages of development is given in Fig. 1. The depth images of Fig.1 were generated from 3D full reconstruction of the seedlings. Shortly, we used a multicamera setup designed for high-throughput phenotyping of seedlings of Fig. 2 and described in $[\square, \mathbb{\square}]$. The seedling is surrounded by different cameras, observing the object from different perspectives. The system can deal with a variable number of cameras. The quality of the 3D reconstruction generally improves with the number of cameras. However, as the gain in quality gets smaller with higher number of cameras and the memory load on the computer increases linearly with the number of cameras, a trade-off between accuracy and efficiency needs to be made. In our experiments, we used 10 cameras as an optimum of the trade-off. The silhouettes of the seedling in the acquired camera images (Fig 2b) are used to reconstruct, with the so-called MARVIN system [ $\square, \mathbb{}, \mathbb{}$ ], the object in 3D through a shapefrom-silhouette method (Fig. 2c). The tomato seedlings chosen in our experiment mostly grow along a vertical axis and leaves are almost positioned horizontaly, i.e. perpendicularly to the vertical axis. The top view depth image generated from the 3D reconstruction of the seedlings is taken along this vertical axis, i.e. the main stem, and the grey levels correspond to the vertical coordinates of each pixels with a millimeter resolution, zero being the ground. The resulting depth images could also be acquired directly with depth imaging systems. In our case the $3 \mathrm{D}$ reconstruction simply serves as ground truth to visually count the leaves. The tomato seedling corresponds to a phenotyping computer vision scene where a single top view image already captures a lot of information on the shoot of the plant specially concerning the counting of the number of leaves as demonstrated in this report.
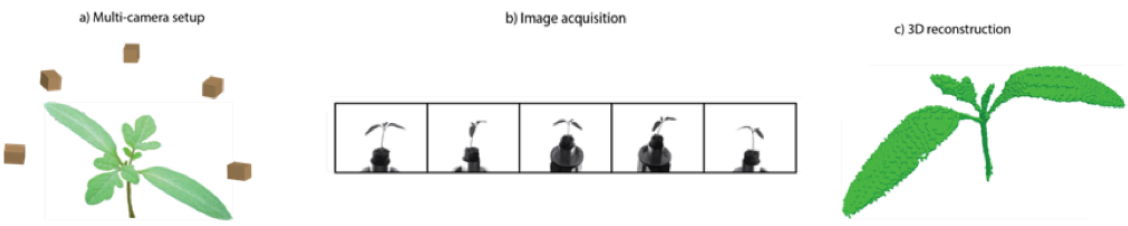

Figure 1: An overview of 3D-reconstruction and segmentation pipeline. a) The plant is observed by multiple cameras (10 used in the experiments). b) Each camera provides an image from which the silhouette of the plant is determined. c) Using a shape-from-silhouette method, the 3D shape of the plant is reconstructed.

\section{Counting leaves algorithm}

The basic idea of the proposed counting leaves algorithm results from the observation of Fig. 3 where the multiscale frequency analysis of Eq. (1) is performed on the top view depth images of tomato seedlings with 2, 3, 4, 5, 6 leaves. As the number of leaves increases, the fit of the averaged power spectrum $D S P(f)$ to a power-law progressively increases. This is visible in Fig. 3 where in the column corresponding to seedlings with 2 leaves the fit is almost 

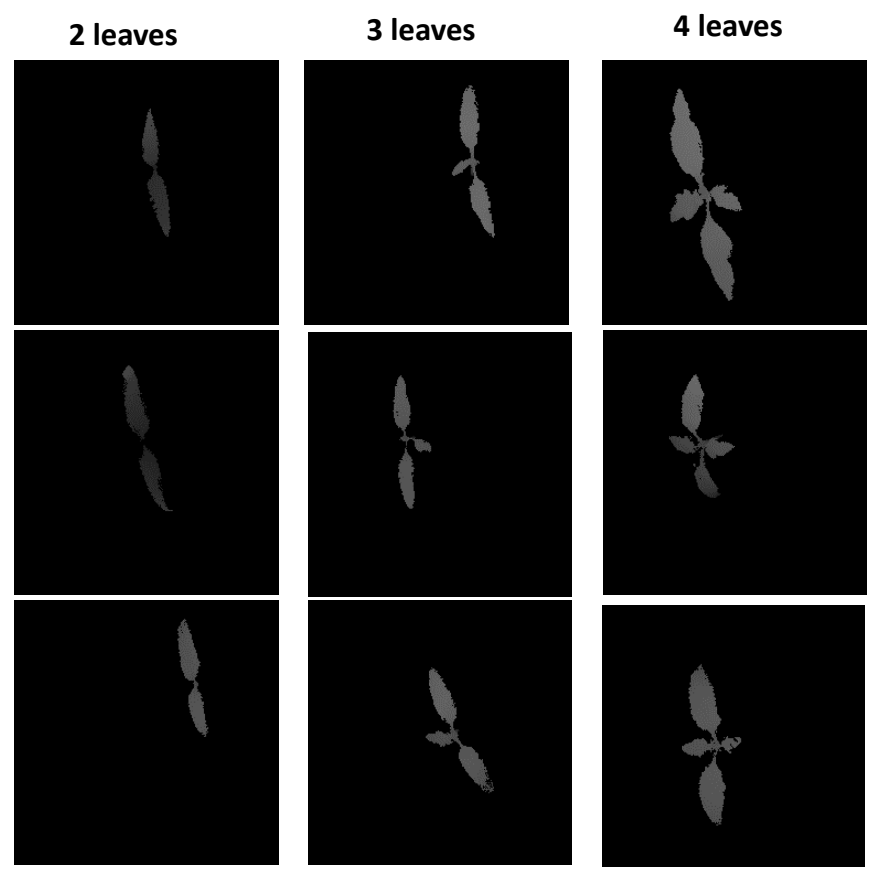

Figure 2: Depth maps from top view of tomato seedlings with 2 leaves (first column), 3 leaves (second column), 4 leaves (third column).

never on the experimental points while for 3 leaves the fit is on the experimental on the range $\log (f)$ between 1000 and 100 and for 4 leaves on the range between 1000 and 50. This trend is not surprising since for a large number of leaves a pure power-law has been demonstrated theoretically in the so-called dead leaves model $[\boldsymbol{\theta}, \square]$. We therefore propose a two steps process. First, in a supervised step, the multiscale frequency analysis of Eq. (1) is performed on a set of seedlings of known number of leaves. A linear fit is realized on $\log (\operatorname{DSP}(f))$ and the standard deviation between the fit and the experimental data is computed. We choose not to include frequency 0 in the fit since it includes information on the size of the surface captured by the depth image and we want to focus on shape. The standard deviation of the fit is then averaged for 10 seedlings of known number of leaves. The values are given in Table 1 where the standard deviation values are clearly distinct. Then, in a second step, this table is used as calibration reference. For a depth image of a tomato seedling with unkown number of leaves (in the range 2 to 6 leaves), the same standard deviation coefficient is computed and the number of leaves is associated to the corresponding closests standard deviation of reference in Table 1 . This procedure has been tested on 50 tomato seedlings (10 for each number of leaves in the range 2 to 6 leaves) and the results are given in Table 2. With this approach, the number of leaves are estimated without having to segment each individual leaves, simply by quantifying the distribution of the spatial frequencies in the depth images in the way they depart from a power-law signature. 
2 leaves
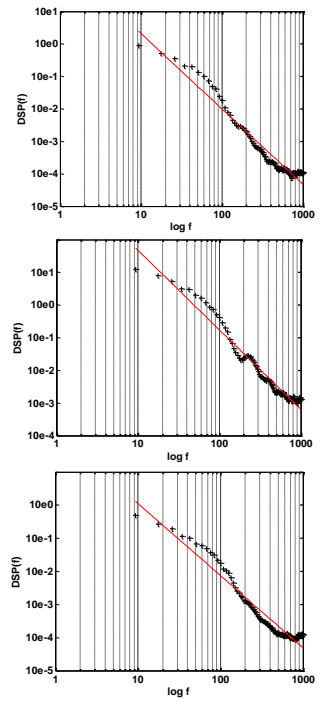

3 leaves
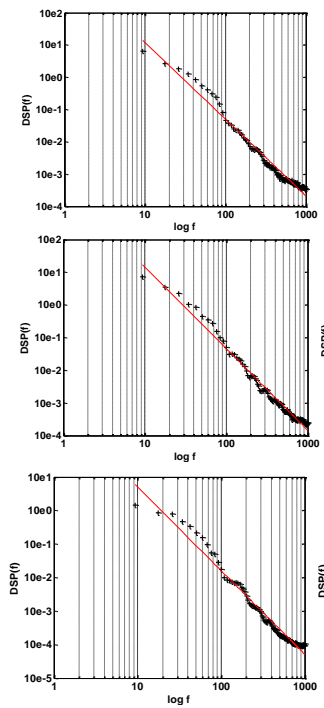

4 leaves
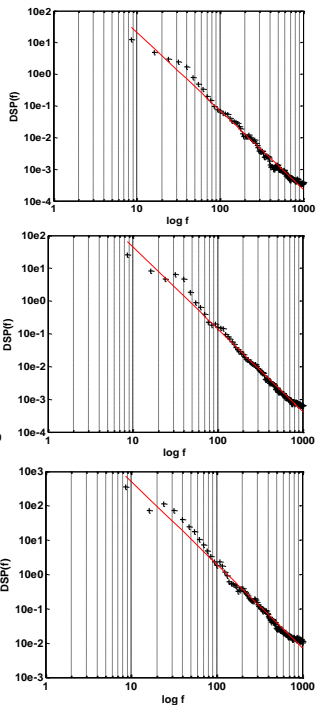

Figure 3: Averaged power spectrum $D S P(f)$ of Eq. (1) computed on the depth images of Fig. 2. The red solid line is the linear fit on a loglog scale of $D S P(f)$.

\section{Discussions}

We now discuss the robustness and limitations of the counting leaves algorithm presented in the previous section. We tested our counting algorithm on plant of the same species at the same stages of development but under environmental conditions which make larger leaves. The results of the counting are excellent (100\% like in Table 1 for smaller leaves with 2, 3 and 4 leaves). To explain this, let us note that seedlings of the same species display the same shape at a given stage of development. A global homothetical change of the size of the plant preserving its shape only modifies the value of $\operatorname{DSP}(f)$ at $f=0$ (not included for this purpose in the analysis) but gives the same evolution of the $\operatorname{DSP}(f)$. This robustness toward the size of the seedling is not surprising since, the counting score is based on the deviation from a power-law which is not sensitive to $\operatorname{DSP}(0)$. We also tested our counting algorithm on plant of different species at the same stages of development. The counting algorithm would be expected to work if the species had the same shape of leaves. But it will not suprisingly fail to count leaves correctly if the leaves have very different shapes from the plant taken as reference. To illustrate this, we picked images of Arabidopsis Thaliana from the CVPPP challenge (http://www.plant-phenotyping.org/CVPPP2014-challenge). Leaves of Arabidopsis Thaliana are much more circular than leaves of the tomato seedlings. Our algorithm failed to count the number of leaves on this data set. The reason is given in Fig. 4. With leaves modeled as ellipses with small eccentricity (less than 0.9 ) the deviation of the average power spectrum from a power law is already very small for a single leaf. Therefore, the smoothing due to the presence of new leaves is very small. As visible in Fig. 4, the 


\begin{tabular}{|l|c|}
\hline Number of leaves & average RMS \\
\hline \hline 2 & 8.5 \\
3 & 7.5 \\
4 & 6.7 \\
5 & 5.9 \\
6 & 5.4 \\
\hline
\end{tabular}

Table 1: Reference standard deviations between the averaged power spectrum $\operatorname{DSP}(f)$ and the linear fit associated to a number of leaves in the counting leaves algorithm. Each reference value is obtained from averaging the standard deviation on 10 seedlings of each class.

\begin{tabular}{|l|c|}
\hline Number of leaves & Number of good classifications \\
\hline \hline 2 & 10 out of 10 \\
3 & 10 out of 10 \\
4 & 10 out of 10 \\
5 & 9 out of 10 \\
6 & 7 out of 10 \\
\hline
\end{tabular}

Table 2: Test of the counting leaves algorithm realized on images of tomato seedlings similar to Fig. 2 with 10 seedlings of each class of number of leaves in the range 2 to 6 .

departure from a power-law is large for elongated leaves. Therefore our algorithm works with elongated leaves such as the one displayed by tomato seedlings in Fig. 2, but will, not surprisingly, fail to count almost circular leaves.

In Table 2, we count the elongated leaves of tomato seedlings correctly only from 2 to 5. As second discussion point, one could wonder why we start counting at 2 . The first reason is biological. The very first leaf which appears when the plant starts its photosynthetic activity is in fact a couple of identical leaves called cotyledons. The second reason is that since these two leaves are identical, they only differ by a translation and rotation. Consequently, the averaged power spectrum of 1 cotyledon is equal to the average power spectrum of 2 cotyledons. Therefore, the deviation from the power-law signature is not affected by the exact replication of a leaf. Our counting algorithm works because when the new leaves appear, they have different sizes than the older leaves. Smaller, i.e. younger leaves will enrich the averaged power spectrum in high spatial frequencies while larger, i.e. older, leaves will relatively contribute to lower spatial frequencies. One could now wonder why our approach fails to go further than counting up to 6 . We applied our algorithm on a plant with 10 leaves as illustrated in Fig. 5. At the stage of development of Fig. 5 some occlusions are present from top view. These occlusions contribute to smooth the average power spectrum. Consequently, as the number of leaves increases, the averaged power spectrum $D S P(f)$ tends to a power-law. Therefore, the evolution of the standard deviation of the experimental data to a power-law will tend to zero independently of the number of leaves for the regime of large number of leaves as expected in the dead leaves fractal model $[$, $\square]$. Our algorithm is thus limited to counting small number of leaves as demonstrated in Table 2. 

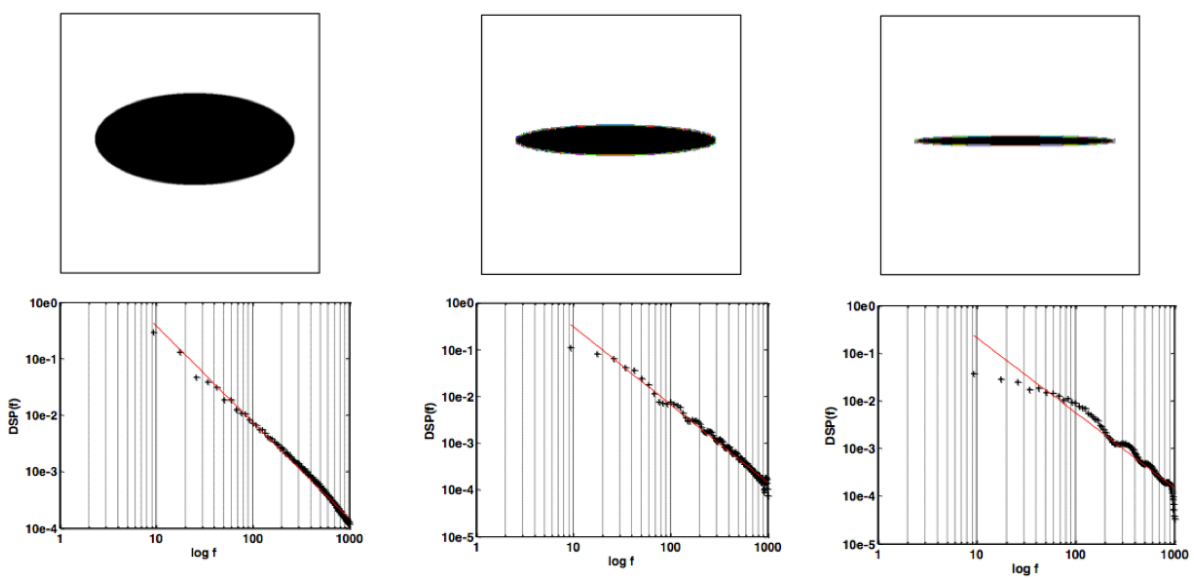

Figure 4: Averaged power spectrum $\operatorname{DSP}(f)$ of Eq. (1) computed on ellipses with various eccentricty taken as model of synthetic leaves. Left column, eccentricity of 0.9 , middle 0.99 and right 0.999 . The red solid line is the linear fit on a loglog scale of $D S P(f)$.

\section{Conclusion and perspectives}

We have introduced a supervised classification algorithm based on multiscale frequency analysis which is able to count elongated leaves from 2 to 6 at early stages of seedling development imaged from top view with depth imaging. This corresponds to a rather limited number of count available but the computer science interest of this approach is that it does not require the segmentation of the shoot nor the actual detection of each individual leaf. Also from an agronomical point of view, the 2 to 6 leaves corresponds to a 10 to 12 days of growth. This corresponds to the stage used by all the breeders for quality assessment of seed batch and breeding purposes to estimate the quality of a new variety. This makes our approach possibly very useful for such industrial tests.

In this report we counted leaves from the average power spectrum computed along the line and column of the depth images. We now shortly discuss the possibility to extract other informations of biological interest with Fourier transform of top view images of mono-axial plant with depth imaging. Leaves on young seedlings are positioned horizontaly. Because of the regular replication at the basis of plant growth, these leaves are positionned at periodic heights. Consequently, an average Fourier transform computed along the vertical axis of a depth image can produce an estimation of the spatial frequency of replication of the leaves. We tested this idea on the plant of Fig. 5 where a principal spatial frequency appears at 1 leaf every $4 \mathrm{~cm}$. This is again interesting since this information is obtained without any segmentation or leaf detection. Also, another dimension where a Fourier transform could be used on depth images from top view is the estimation of diurnal leaf growth. This biological process, produces oscillations of the leaves which can be estimated from computer vision

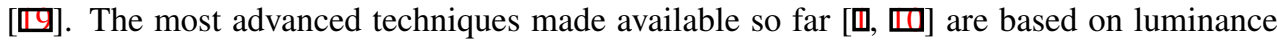
imaging. Depth images give better contrasts and recording series of top view with depth images would enable to sense the evolutions of the shape of the plant. Temporal Fourier transform computed along pixels in the time series would carry information on these temporal evolutions again without the need to resort to individual segmentation or detection of the 
leaves.
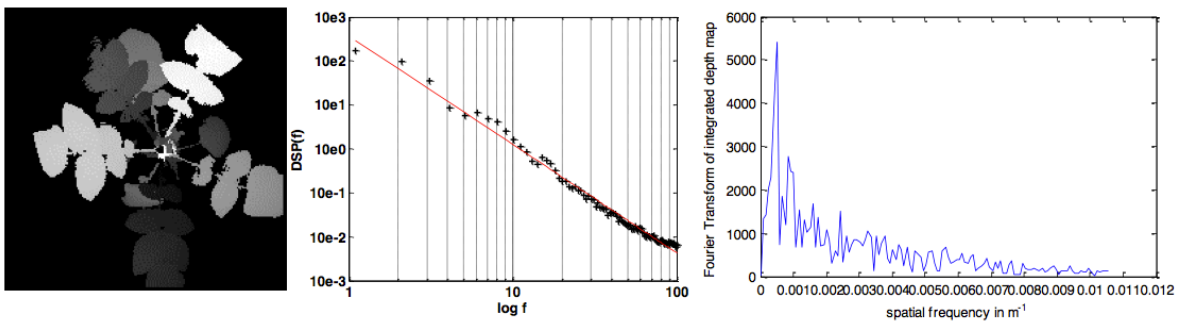

Figure 5: Top view of a tomato seedling with 10 leaves (left), (middle) corresponding averaged power spectrum $D S P(f)$ of Eq. (1) and (right) vertical power spectrum.

\section{References}

[1] R. Bours, M. Muthuraman, H. Bouwmeester, and A. van der Krol. Oscillator: A system for analysis of diurnal leaf growth using infrared photography combined with wavelet transformation. Plant methods, 8(1):29, 2012.

[2] F. Chapeau-Blondeau, J. Chauveau, D. Rousseau, and P. Richard. Fractal structure in the color distribution of natural images. Chaos, Solitons, Fractals, 42:472 - 482, 2009.

[3] J. Chauveau, D. Rousseau, and F. Chapeau-Blondeau. Fractal capacity dimension of three-dimensional histogram from color images. Multidimension Systems Signal Processing, 21:197 - 211, 2010.

[4] J. Chauveau, D. Rousseau, P. Richard, and F. Chapeau-Blondeau. Multifractal analysis of three-dimensional histogram from color images. Chaos, Solitons, Fractals, 43:57 $67,2010$.

[5] Y. Chéné, D. Rousseau, P. Lucidarme, J. Bertheloot, V. Caffier, P. Morel, E. Belin, and F. Chapeau-Blondeau. On the use of depth camera for 3D phenotyping of entire plants. Computers and Electronics in Agriculture, 82:122-127, 2012.

[6] Y. Chéné, É. Belin, D. Rousseau, and F. Chapeau-Blondeau. Multiscale analysis of depth images from natural scenes: Scaling in the depth of the woods. Chaos, Solitons and Fractals, 54:135 - 149, 2013.

[7] Y. Chéné, E. Belin, F. Chapeau-Blondeau, V. Caffier, T. Boureau, and D. Rousseau. Anatomofunctional bimodality imaging for plant phenotyping: An insight through depth imaging coupled to thermal imaging. Plant Image Analysis: Fundamentals and Applications, page 179, 2014.

[8] P. Ferraro, C. Godin, and P. Prusinkiewicz. Toward a quantification of self-similarity in plants. Fractals, 13:91 - 109, 2005.

[9] Y. Gousseau and F. Roueff. Modeling occlusion and scaling in natural images. SIAM Journal of Multiscale Modeling and Simulation, 6:105-134, 2007. 
[10] K. Greenham, P. Lou, S. Remsen, F. Hany, and C. McClung. Trip: Tracking rhythms in plants, an automated leaf movement analysis program for circadian period estimation. Plant Methods, 11(1):33, 2015.

[11] A. B. Lee, D. Mumford, and J. Huang. Occlusion models for natural images: A statistical study of a scale-invariant dead leaves model. International Journal of Computer Vision, 41:35-59, 2001.

[12] Z. Moravek and J. Fiala. Fractal dynamics in the growth of root. Chaos, Solitons, Fractals, 19:31 - 35, 2004.

[13] F. Golbach G. Otten H. Gerlich H. J. van de Zedde N. Koenderink, M. Wigham. Marvin: High speed 3d imaging for seedling classification. Seventh European Conference on Precision Agriculture, pages 279 - 286, 2009.

[14] P. Prusinkiewicz and A. Lindenmayer. The algorithmic beauty of plants. Berlin: Springer, 2004.

[15] D. Rousseau, Y. Chéné, E. Belin, G. Semaan, G. Trigui, K. Boudehri, F. Franconi, and F. Chapeau-Blondeau. Multiscale imaging of plants: current approaches and challenges. Plant methods, 11(1):6, 2015.

[16] D. L Ruderman. The statistics of natural images. Network: Computational Neural Systems, 5:517 - 548, 1994.

[17] D. L Ruderman and W. Bialek. Statistics of natural images: Scaling in the woods. Physical Review Letters, 43:814 - 821, 1994.

[18] D. Da Silva, F. Boudon, C. Godin, and H. Sinoquet. Multiscale framework for modeling and analyzing light interception by trees. Multiscale Model Simulation, 7:910 - 933, 2008.

[19] Andrew J Tindall, Jade Waller, Mark Greenwood, Peter D Gould, James Hartwell, and Anthony Hall. A comparison of high-throughput techniques for assaying circadian rhythms in plants. Plant Methods, 11(1):32, 2015.

[20] H. J. van de Zedde. Rapid 3d reconstruction of plants and the benefits for phenotyping and robotics. 3rd International Plant Phenotyping Symposium, Chennai - India, February 18, 2014. 